\title{
Proapoptotic protein Smac mediates apoptosis in ovarian cancer cells when treated with carpachromene
}

\author{
Yunjing Song ${ }^{1}$, Jian Wang ${ }^{2}$, Chunnian Zhang ${ }^{1}$, Ying Yu ${ }^{1}$, Hong Cai ${ }^{1}$
}

${ }^{1}$ Department of Gynaecology, Ganzhou People's Hospital, Ganzhou, Jiangxi, China 2Pathology Department, Ganzhou Cancer Hospital, Ganzhou, Jiangxi, China

Submitted: 16 October 2021; Accepted: 1 November 2021

Online publication: 7 November 2021

Arch Med Sci

DOI: https://doi.org/10.5114/aoms/143512

Copyright $\odot 2021$ Termedia \& Banach

\section{Abstract}

Introduction: We investigated carpachromene in cytotoxicity studies against a common human ovarian cancer cell line, i.e. SW 626, in vitro. Interestingly, we obtained significantly good results in the study. Also, we performed enzyme inhibition and molecular docking studies.

Material and methods: For investigating the antioxidant properties of carpachromene, the DPPH test was used in the presence of butylated hydroxytoluene as the positive control. Cell viability of carpachromene was very low against the common human ovarian cancer cell line SW 626 without any cytotoxicity on a normal cell line. To compare the biological activities of molecules, the enzymes used were $\alpha$-glucosidase, acetylcholinesterase, respectively. Finally, calculations were made using the molecular docking method to compare the biological activity of the carpachromene molecule. We then examined whether the release of Smac is necessary for apoptosis in ovarian cancer cells using the SW 626 cell line. We first examined mitochondrial and cytosolic Smac levels after carpachromene treatment.

Results: Carpachromene inhibited half of the DPPH molecules in the concentration of $103 \mu \mathrm{g} / \mathrm{ml}$. Maybe significant anti-human ovarian cancer potential of carpachromene against common human ovarian cancer cell lines is linked to their antioxidant activities. Following the docking calculations, the properties of the carpachromene molecule were examined by ADME/T analysis in order to be used as a drug in the future. In addition, the anti-oxidant properties of the molecules were examined in both gas and water phases with the HF/6-31g basis set with the Gaussian software program. It was found that exposure of ovarian cancer cells to carpachromene decreased mitochondrial Smac and increased cytosolic Smac levels in a time-dependent fashion. The results showed a decrease in Smac expression, as confirmed by Western blot. Silencing of Smac significantly inhibited carpachromene-induced caspase-3 cleavage and attenuated apoptosis in these cells. Moreover, overexpression of a Smac heptapeptide (Smac-N7) enhanced carpachromene-induced cell death. Conclusions: According to the above findings, carpachromene may be administered for the treatment of several types of human ovarian cancer in humans.

Key words: carpachromene, human ovarian cancer, Smac, caspase-3 cleavage.

\section{Introduction}

Alpha-glucosidase inhibitors (AGIs; acarbose, miglitol, voglibose) are widely used in the treatment of patients with type 2 diabetes. AGIs delay the absorption of carbohydrates from the small intestine and thus

\author{
Corresponding author: \\ Hong Cai \\ Department of Gynaecology \\ Ganzhou People's Hospital \\ Ganzhou 341000 \\ Jiangxi, China \\ E-mail: CH10262020@163. \\ com
}


have a lowering effect on postprandial blood glucose and insulin levels [1, 2]. Acetylcholinesterase (AChE; EC 3.1.1.7) creates optimum conditions for the electron carriers by removing the chemicals that accumulate in the nerve end over time. There is no definitive treatment method for Alzheimer's disease as in Parkinson's disease. The current treatment methods are only aimed at minimizing the effects of the disease or improving the quality of life. For this purpose, AChE inhibitors (donepezil, rivastigmine) are widely used in order to ensure a better quality of life for patients $[3,4]$.

Many recent studies have shown that when theoretical and experimental studies are carried out together, it has been observed that the research has increased quality. Both the results of the theoretical studies and the results of the experimental studies were found to be in great harmony with each other [5-8]. In the theoretical studies, both the anti-oxidant properties and biological activities of the molecule studied were examined by calculations.

One of the best and common methods used to compare the biological activities of molecules is molecular docking. In order to compare the biological activities of the carpachromene molecule, calculations were made against many enzymes by molecular docking calculations [9]. As a result of these calculations, many parameters related to the carpachromene molecule were obtained. The numerical values of these parameters give information about many properties of the molecule. After these calculations, ADME/T (absorption, distribution, metabolism, excretion and toxicity) analysis was performed to examine the molecule's potential to be used as a drug. As a result of $A D M E / T$ analysis, many parameters were found. If the numerical values of these parameters are within certain ranges, this molecule can be used as a medicine.

Calculations were made to examine the antioxidant properties of the carpachromene molecule. The most important reason for examining these anti-oxidant properties is that some molecules in the human body turn into high-activity free radicals during biochemical reactions [10]. The fact that these free radicals have high activity to the cells and organs in the human body causes great damage. It causes many chronic diseases such as cancer and heart diseases at the beginning of this damage. By inhibiting these free radicals, it is intended to minimize this damage [11-17]. Therefore, the anti-oxidant property of this carpachromene molecule has been studied.

We also investigated carpachromene in cytotoxicity studies against a common human ovarian cancer cell line, i.e., SW 626, in vitro. Interestingly, we obtained significantly good results in the study. Also, we performed enzyme inhibition and molecular docking studies.

\section{Material and methods}

\section{Determination of antioxidant activities of carpachromene}

In this method, first $0.3 \mathrm{ml}$ of the sample solution was poured into a test tube, then $9 \mathrm{ml}$ of the DPPH methanolic solution was added. The contents of each tube were thoroughly mixed with the vortex. After $30 \mathrm{~min}$, at room temperature and in the dark, their absorbance was read at $517 \mathrm{~nm}$ using a UV/Vis spectrophotometer against a methanol-containing blank. In this method, BHT was used as a positive control. According to the mentioned mechanism, the higher the antioxidant power of the sample, the yellower the color of the resulting solution will be.

The following formula was used to determine the antioxidant properties of carpachromene: Inhibition $(\%)=($ Sample A/Control A $) \times 100$.

Excel and Bio Data Fit 1.02: Data Fit For Biologists software was used to calculate the $I_{50}$ and to compare the antioxidant effects of these extracts with the standards and negative control and plotting [18].

\section{Gene silencing with small interfering RNAs and plasmids}

Small interfering RNA (siRNA) oligonucleotides were purchased from Dharmacon (Lafayette, CO) with sequences targeting Bax (5'- AACUGAUCAGAACCAUCAUGG-3'), Smac (5'-AACCCUGUGUGCGGUUCCUAU-3'), and p53 (5'-CGG-CAUGAACCGGAGGCCCAU-3'). For Bax shRNA construction, the Bax siRNA was cloned into the pSilencer 2.1-U6 hygroplasmid. The constitutively active Akt1 construct HA-PKB-T308D/S473D was obtained as described previously. Smac and XIAP constructs were generated by RT-PCR from total RNA isolated from A2780/CP cells (cisplatin-resistant version of the A2780 cells) and cloning of the RT-PCR products into the pFLAG-CTC vector (Sigma).

\section{Determination of anti-human ovarian cancer effects of carpachromene}

The process of controlled culture of prokaryotic or eukaryotic cells in a filtered or unfiltered flask or cell culture plate by a suitable culture medium is called. This term is mostly used for culturing multicellular cells. Special culture media are used to culture cells. The cells are usually cultured at $37^{\circ} \mathrm{C}$ in equipment such as $\mathrm{CO}_{2}$ incubators. Cell culture should be performed under aseptic (disinfected) conditions because the growth of these cells is much slower than the growth of bacteria 
and yeasts and there is a possibility of contamination of the culture medium. Antibiotics such as penicillin, streptomycin, or gentamicin are sometimes used to stop the growth of bacteria. In order for cells to proliferate well in culture medium, their density in culture medium must be low. For this purpose, the cells should be passed to fresh culture medium from time to time. One of the goals of cell culture is to study cells in terms of how they grow, their nutritional needs, and the reasons they stop growing, each of which can have a profound effect on the morphology of the cells we see under a microscope. Therefore, to study the cell growth cycle, develop methods to control the growth of cancer cells and modulate the expression of genes, it is necessary to cultivate these cells in an external environment [19-24].

With the help of cell culture, cells can be prepared that are in different stages of differentiation and can be differentiated into other cells with the help of hormones and growth factors. With the help of cell culture, homogeneous cells can be prepared and intracellular activities such as DNA replication, DNA transcription synthesis, RNA and protein synthesis and other details related to metabolism can be studied. It is also possible to examine the subsequent events and intracellular currents, such as the displacement of these complexes, the type of intracellular messages, and how the messages are transmitted, after connecting different molecules to the corresponding membrane receptor. The cultured cells can be stored frozen at very low temperatures. Such conditions will maintain the growth rate or genetic composition of these cells and they can be thawed and used again at the appropriate time. This prevents aging of cells, while it is currently not possible to prevent the aging of animals. When working with laboratory animals, systemic changes due to the effect of the animal's natural homeostasis or the stress of the experiments on the results should be considered, while the use of cell culture eliminates this problem. In addition, standardizing laboratory tests is easier and more practical than tests on living organisms. In laboratory environments, it is much easier to control the physical and chemical factors in the living environment of cells, including acidity, heat, osmotic pressure, and the pressure of gases such as oxygen and carbon dioxide. Cells that are taken directly from the individual are known as primer cells and have a limited lifespan. Most cells have a limited lifespan, except for those taken from a tumor. An immortal cell line can proliferate indefinitely by creating a random or targeted mutation (such as artificial expression of the genes) and be established as a representative of specific cell types [19-24].
In the present experiment, the human ovarian cancer cell line SW 626 and the human normal cell line (HUVEC) were used to study the cytotoxic and anticancer potential of carpachromene against human ovarian cancer using a common cytotoxicity test i.e., MTT assay in in vitro conditions.

The cells were cultured in medium (RPMI 1640 = Roswell Park MemoryL Institute1640) with 10\% FBS combined with penicillin and streptomycin antibiotics in an incubator containing $5 \% \mathrm{CO}_{2}$ in a flask (T25). After three passages for purification, the cells were used to perform the next steps. Cell count and the number of viable cells were determined with a hemocytometer slide using trypan blue. Evaluation of the cytotoxic effect of carpachromene was performed by the modified 3-(4,5-dimethylthiazol-2-yl)-2,5-diphenyl-2H-tetrazolium bromide (MTT) colorimetric test. In this method, MTT, which is yellow, is converted to insoluble and formazan purple dye by dehydrogenase enzymes in the mitochondria of active cells. The adsorption of this compound can be measured after dissolving at 570-540 nm. After 2 days and covering the flask bottom with cells, the cell layer adhering to the flask bottom was isolated enzymatically using trypsin-EDTA (5\%) (tetraacetic acid ethylenediamine); after transfer to sterile test tubes, it was centrifuged at $2000 \mathrm{rpm}$ for $10 \mathrm{~min}$. The cells were then resuspended in a fresh culture medium with the help of a Pasteur pipette and cell suspension $\left(10^{6} \mathrm{ml} / \mu \mathrm{g}\right)$ was prepared from them. $40 \mu \mathrm{l}$ of this cell suspension (equivalent to $10^{4} \times 4$ cells) was poured into 96-well plate flat-bottomed wells (for cell culture). Then the final volume of each well with $10 \%$ FBS medium reached $200 \mu \mathrm{l}$. The first row containing cell suspension was considered as a negative control (control). After incubation for 18-24 $\mathrm{h}$ to remove cells from the stress caused by trypsinization, the supernatant was removed slowly and carefully. A new medium was added to all rows with different concentrations of carpachromene (only a new medium was added to the negative and positive control rows), so that the diluted carpachromene with concentrations of $1-1000 \mu \mathrm{g} / \mathrm{ml}$ was added to the third to sixth rows, respectively, and the plate was incubated in $\mathrm{CO}_{2}$ for 48,24 and $72 \mathrm{~h}$. After the incubation time, the plate was taken out of the incubator and $20 \mu$ l of MTT (Sigma) was added to all wells, and incubated for $3 \mathrm{~h}$. The supernatant was then gently removed and $100 \mu$ l of DMSO was added to the wells and pipetted to dissolve the formazan crystals. The amount of light absorption (OD) according to the intensity of the blue color of formazan at $540 \mathrm{~nm}$ was read by an ELISA reader. To convert OD to the percentage of living cells, the following formula was used and the percentage 
of living cells at each concentration was calculated after 48, 24 and 72 h [19-24]: Cell viability (\%) $=($ Sample A/Control A $) \times 100$.

The concentration of the tested compounds that reduced the percentage of living cells by half was considered as $\mathrm{IC}_{50}$ (the half maximal inhibitory concentration) [19-24].

\section{Qualitative measurement}

At least three independent replications were performed for each data and the result was presented as mean \pm SD. Data statistical analysis was done with SPSS software version 22 and one-way ANOVA and Duncan tests. Significance was considered at the level of $p \leq 0.05$.

\section{Docking study}

In this study, molecular docking calculations to compare the biological activities of molecules were performed using the Maestro Molecular modeling platform (version 12.2) by Schrödinger. Proteins and molecules must be prepared for calculations. In the calculations, a different process is performed for the molecules at each stage. Firstly, the Gaussian software program was used to obtain optimized structures of molecules, which created files with the extension *.sdf using these structures. Using these files, all calculations were made with the Maestro Molecular modeling platform (version 12.2) by Schrödinger, LLC [25]. The Maestro Molecular modeling platform (version 12.2) by Schrödinger comprises many modules. In the first module used, the protein preparation module $[26,27]$ was used to prepare the proteins for calculations. The crystal structures of these proteins have been downloaded from the Protein Data Bank site. These proteins were initially minimized and water molecules in their crystal structures were removed. In the next step, the active regions of the proteins were determined for calculations, in which the proteins in this active region were given freedom of movement. Therefore, these proteins were enabled to interact with molecules more easily. In the next step, the LigPrep module $[28,29]$ was used to prepare the working molecules for calculations.

Calculations were performed to find high energy isomers in physiological pH values of new cyanopyridine derivatives containing 3D structures and the correct protonation conditions. In the next step, the prepared proteins and molecules were docked with each other. The Glide ligand docking module [30] was used for this step. The enzymes used were $\alpha$-galactosidase ( $\alpha$-Gly) (1T0O) [31], acetylcholinesterase (4MOE) (AChE) [32-34], respectively. In this module, the OPLS3e method was used in all calculations for docking calcula- tions of molecules and proteins. The numerical values of many parameters obtained as a result of molecular docking calculations using this module were used. After the docking calculations, ADME/T analysis (absorption, distribution, metabolism, excretion and toxicity) was performed to examine the molecule's ability to be a drug in the future. The Qik-prop module [35] of the Schrödinger software was used for ADME/T analysis.

\section{Gaussian study}

Theoretically, the process of comparing the anti-oxidant activities of molecules requires calculating more than one thermodynamic parameter. These thermodynamic parameters are bond dissociation enthalpy (BDE), proton dissociation enthalpy (PDE), proton affinity (PA), ionization potential (IP) and electron transfer enthalpy (ETE) [36-40].

In this study, 3 mechanisms were examined in the anti-oxidant study. The first one is the hydrogen atom transfer mechanism (HAT):

$$
R^{\bullet}+A H \rightarrow R H+A^{\bullet}
$$

In this mechanism, the free radical molecule extracts a hydrogen from the anti-oxidant and converts the anti-oxidant into its free radical form. To calculate the reactivity of the compound, it is necessary to calculate the bond dissociation enthalpy (BDE) of the $\mathrm{A}-\mathrm{H}$ bond.

The second mechanism is single electron transfer-proton transfer (SET-PT). This mechanism consists of two consecutive reactions. In the first one, the removal of the electron from the $\mathrm{AH}$ occurs immediately after the proton transfer.

$$
\begin{gathered}
R^{\bullet}+A H \rightarrow R^{-}+A H^{\bullet} \\
A H^{\bullet} \rightarrow H^{+}+A^{+}
\end{gathered}
$$

These two mechanisms are ionization potential (IP) and proton dissociation enthalpy (PDE) from the $A H^{+}$cation radical, respectively.

The mechanism of the third and last reaction is again in two stages. These two mechanisms are called the SPLET (sequential proton loss electron transfer) mechanism.

$$
\begin{gathered}
A H \rightarrow A^{-}+H^{+} \\
A^{-}+H^{+}+R^{\bullet} \rightarrow A^{\bullet}+R H
\end{gathered}
$$

The reaction enthalpy of the first reaction is the proton affinity (PA) of the anion. The reaction enthalpy of the second reaction is electron abstraction, namely electron transfer enthalpy (ETE). The enthalpies of the reactions for these five reactions are calculated from the formulas below.

$$
\begin{gathered}
B D E=H\left(A^{\bullet}\right)+H\left(H^{\bullet}\right)-H(A H) \\
I P=H\left(A H^{+\bullet}\right)+H\left(e^{-}\right)-H(A H) \\
P D E=H\left(A^{\bullet}\right)+H\left(H^{+}\right)-H\left(A H^{+\bullet}\right)
\end{gathered}
$$




$$
\begin{aligned}
& P A=H\left(A^{-}\right)+H\left(H^{+}\right)-H(A H) \\
& E T E=H\left(A^{\bullet}\right)+H\left(e^{-}\right)-H\left(A^{-}\right)
\end{aligned}
$$

To calculate the numerical values in these formulas, GaussView 5.0.8 and Gaussian09 AS64L-G09RevD.01 [8, 41] package programs were used. Calculations of anti-oxidant molecules were performed using the Hartree-Fock (HF) method $[42,43]$ with the $6-31++G(d, p)$ basis set in the gas and water phase.

\section{Assessment of enzyme activities}

Ellman's method [44] was used with slight modifications. A disposable cuvette was filled with $0.4 \mathrm{ml}$ of $0.4 \mathrm{mg} / \mathrm{ml}$ DTNB, $25 \mu \mathrm{l}$ of AChE solution (0.5 $\mu$ kat in $1 \mathrm{mM}$ acetylthiocholine), $425 \mu \mathrm{l}$ of PBS, $50 \mu \mathrm{l}$ of paraoxon in isopropanol or isopropanol alone $[45,46]$. The reaction was started by adding $100 \mu \mathrm{l}$ of acetylthiocholine chloride in a given concentration for assessment of $\mathrm{K}_{\mathrm{m}}$ and $\mathrm{V}_{\max }$ or $1 \mathrm{mM}$ for toxicological and pharmacological investigations. Absorbance at $412 \mathrm{~nm}$ was measured immediately and after $1 \mathrm{~min}$. Enzyme activity was calculated estimating extinction coefficient $\varepsilon=14,150 \mathrm{M}^{-1} \mathrm{~cm}^{-1}$. The inhibition effect of glucosidase of carpachromene was assessed using the Tao et al. [47] process. First, the phosphate buffer $(\mathrm{pH} 7.4,75 \mu \mathrm{l})$ was combined with $5 \mu \mathrm{l}$ of the sample and $20 \mu \mathrm{l}$ of the $\alpha$-glycosidase enzyme solution prepared in the phosphate buffer $(0.15 \mathrm{U} / \mathrm{ml}, \mathrm{pH} 7.4)$. After pre-incubation, $50 \mu \mathrm{l}$ of p-nitrophenyl-D glycopyranoside ( $p-N P G)$ was applied to the phosphate buffer $(5 \mathrm{mM}, \mathrm{pH} 7.4)$ and re-incubated at $37^{\circ} \mathrm{C}$. At $405 \mathrm{~nm}$ the absorbance of mixtures was reported. Three separate concentrations of carpachromene were used to de-terminate the Ki values [48]. The graphs were then drawn from Lineweaver-Burk [49].

\section{Results and Discussion}

\section{Enzyme results}

Inhibition of metabolic enzymes was investigated, and the results were reported as follows.

Carpachromene was effective in inhibiting AChE as a metabolic enzyme. The $\mathrm{K}_{\mathrm{i}}$ value for AChE was $56.24 \pm 12.32 \mathrm{nM}$ (Table I). Also, the tacrine (TAC) molecule was used as an AChE enzyme control molecule; it had a $K_{i}$ value of $102.67 \pm 11.57$
nM. Carpachromene and TAC IC $C_{50}$ values were: carpachromene (63.87 nM, $\left.r^{2}=0.9809\right)<$ TAC (123.93 nM, $\left.r^{2}=0.9678\right)$ for AChE. AChE inhibitor compound is a neurotoxic molecule capable of causing central, peripheral, or both peripheral and central cholinergic crises. The molecule investigated in the present study can be applied as medicinal products developed to treat myasthenia gravis and $A D$.

On the other hand, carpachromene showed $\mathrm{IC}_{50}$ and $\mathrm{K}_{\mathrm{i}}$ values are $112.98 \mathrm{nM}, r^{2}=0.9881$ and $135.03 \pm 16.15$ (Table I). For the $\alpha$-glucosidase present on cell lining, and the intestine, hydrolyzing monosaccharides are absorbed through the intestine. The results of the $\alpha$-glucosidase assay showed that carpachromene has an effective $\alpha$-glucosidase inhibition profile compared to that of acarbose $\left(\mathrm{IC}_{50}=128.26 \mathrm{nM}, \mathrm{K}_{\mathrm{i}}=141.34 \pm 17.55\right)$ as a standard $\alpha$-glucosidase inhibitor. The inhibition of $\alpha$-glucosidase digestive enzyme is of great importance for the treatment and prevention of diabetes, postprandial glucose levels and hyperglycemia.

\section{Antioxidant capacities of carpachromene}

Free radicals are molecules with a free electron ready to react, and oxygen is produced with some molecules. If many of them are suddenly produced in the body, they react with some parts of the cell, such as DNA and cell membranes, and cause cell damage or even death. Normally, the body's defense system neutralizes these harmless free radicals $[48,49]$. Antioxidants prevent the spread of oxidation chain reactions. Thus, the strength of an antioxidant formed by the contact of an $\mathrm{H}$ atom with a free radical is due to the effect of an antioxidant on the ease with which this $\mathrm{H}$ atom separates from it. Thus, antioxidants can protect cell membranes and various compounds against oxidants in small amounts. Numerous biochemical and physiological processes may cause the production of free radicals. Reactive oxygen species (ROS) include free radicals and radical-free forms. Free radicals include hydrogen peroxide $\left(\mathrm{H}_{2} \mathrm{O}_{2}\right)$, hydroxyl radical $\left(\mathrm{OH}^{\circ}\right)$, and superoxide anion radical $\left(\mathrm{O}_{2}^{-\bullet}\right)$. When the concentration of ROS increases, it can oxidize macromolecules such as proteins, nucleic acids, and membrane lipids,

Table I. Enzyme inhibition results of carpachromene against acetylcholinesterase (AChE) and $\alpha$-glucosidase ( $\alpha$-Gly) enzymes

\begin{tabular}{|lcccccc|}
\hline \multirow{2}{*}{ Compounds } & \multicolumn{7}{c|}{$\mathrm{IC}_{50}$ and $\mathrm{K}_{\mathrm{i}}$ values [nM] } \\
\cline { 2 - 7 } & $\mathrm{AChE}$ & $\boldsymbol{r}^{2}$ & $\alpha$-Gly & $r^{2}$ & AChE & $\alpha$-Gly \\
\hline Carpachromene & 63.87 & 0.9809 & 112.98 & 0.9881 & $56.24 \pm 12.32$ & $135.03 \pm 16.15$ \\
\hline Tacrine & 123.93 & 0.9678 & - & - & $102.67 \pm 11.57$ & - \\
\hline Acarbose & - & - & 128.26 & 0.9013 & - & $141.34 \pm 17.55$ \\
\hline
\end{tabular}




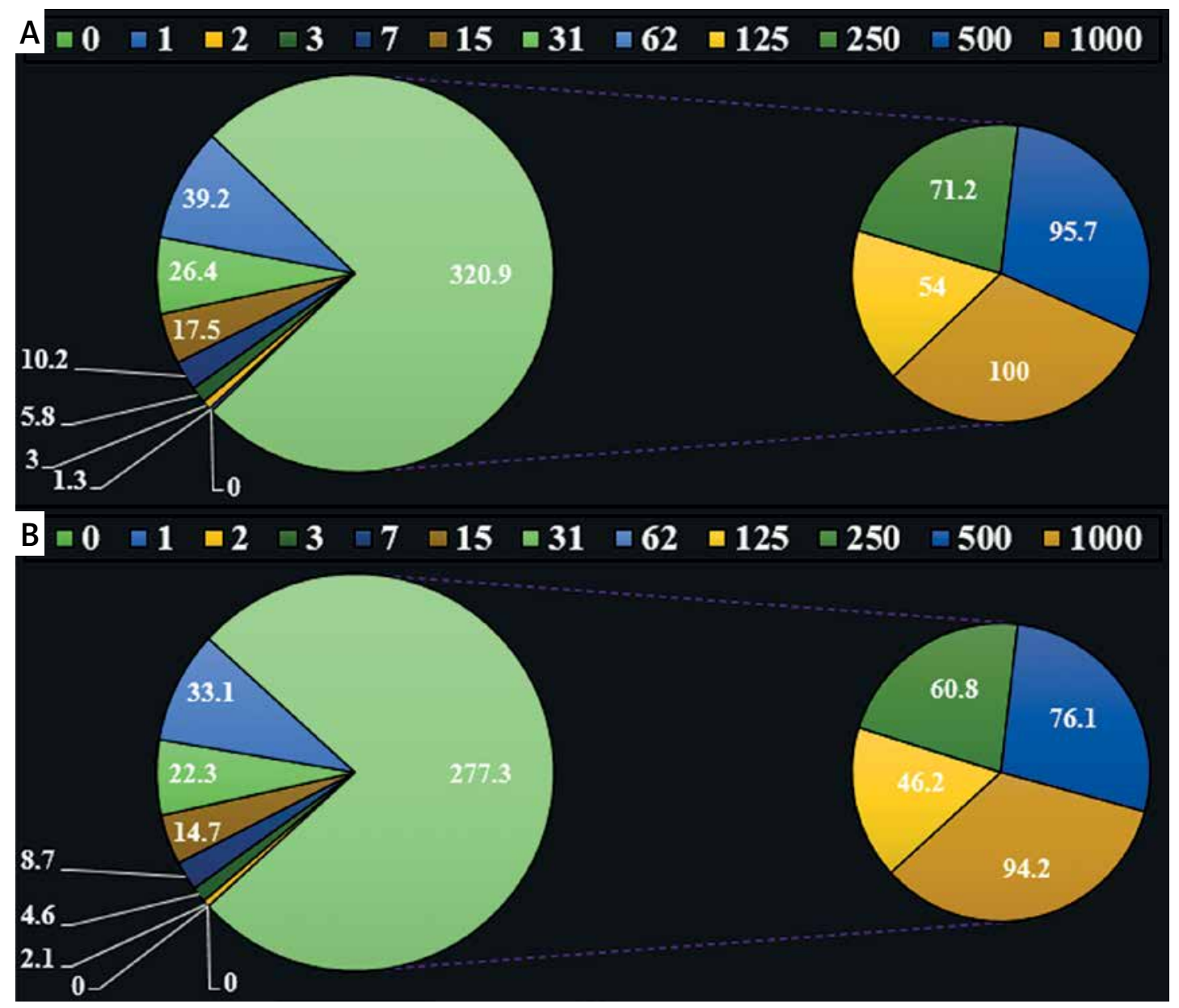

Figure 1. Antioxidant properties of carpachromene (A) and BHT (B) against DPPH. The numbers indicate the percent of free radical (DPPH) inhibition in the concentrations of $0-1000 \mu \mathrm{g} / \mathrm{ml}$ of carpachromene and BHT

resulting in cell damage and possibly "cell and tissue destruction" [43-46]. Natural compounds and molecules reduce the concentration of ROS by producing antioxidants and thus prevent cell damage. Recently, many researchers have paid close attention to natural compounds and molecules and their relationship to their antioxidant properties, and many natural compounds and molecules have been studied for their antioxidant activity.

Now, turning our attention to investigate the bioactivity of carpachromene, a concentration-dependent DPPH radical scavenging effect of carpachromene was observed against $\mathrm{BHT}$ as a reference.

The DPPH process is widely applied to determine the free radical scavenging effect of different antioxidant materials. The DPPH scavenging abilities are known to be due to the hydrogen donating activities of antioxidant materials. When DPPH

Table II. IC 50 of carpachromene and BHT in the antioxidant test

\begin{tabular}{|lcc|}
\hline & Carpachromene & BHT \\
\hline $\mathrm{IC}_{50}[\mu \mathrm{g} / \mathrm{ml}]$ & 103 & 155 \\
\hline
\end{tabular}

results were examined, it was observed that it increased in a dose-dependent manner [24].

The interaction between carpachromene and DPPH might have occurred by transferring electrons and hydrogen ions. The scavenging capacity of carpachromene and BHT at different concentrations, expressed in terms of percentage inhibition, is shown in Figure 1.

In the antioxidant test, the $\mathrm{IC}_{50}$ values of butylated hydroxytoluene and carpachromene were 155 and $103 \mu \mathrm{g} / \mathrm{ml}$, respectively (Table II).

Antioxidant compounds show stronger antioxidant effects against free radical formation in the living system [50]. The antioxidant compounds have excellent redox properties and have a significant role in deactivating free radicals. Previous studies have indicated that flavonoids and phenolic compounds have significant antioxidant properties [51, 52].

\section{Cytotoxicity and anti-human ovarian cancer potential of carpachromene}

Cancer is recognized as one of the leading causes of death in today's society, and several 
drugs have been introduced to treat this disease, but most common cancers are not yet controllable, and this disease imposes huge costs on patients and society. The main factor in the development and progression of cancer has not yet been precisely identified. However, the available data suggest that metabolic disorders in the tissue and immune disorders may be involved in the development and exacerbation of this disease [51, 52]. In addition, metabolic disorders in the production and excretion of oxygen free radicals are important factors affecting cancer cells. Free radicals are destructive compounds that are produced as a by-product by the body's chemical reactions and are destroyed by the body's defense system and enzyme system and antioxidants. However, in cases where the body's metabolic disorders and the production of free radicals are high and they are not destroyed by the neutralizing system, due to their instability, these compounds have a strong tendency to react with a variety of molecules in the body [51]. It is estimated that each cell in the human body is exposed to free radicals 10,000 times a day and DNA strands 5,000 times a day. Damage to cell components includes proteins (genetic disorder), fats (lipid oxidation), and cell membranes (permeability disorder) such that if the damage is not repaired, it leads to disruption of the chemical reaction and normal proteinization of the cell and the formation of harmful compounds and sometimes cancer cells in the body [52]. It is reported that thousands of cancer cells are produced daily in the human body, which are killed by the body's defense system. In some cases, due to dysfunction of the above systems, cancer cells proliferate and conditions for cancer develop in different tissues. According to the above, antioxidants play a vital role in preventing disorders caused by the effects of free radicals and thus the prevention and treatment of cancer. Antioxidants are a wide range of molecular compounds with complex properties that combine with and neutralize free radicals. More than 60,000 types of molecular antioxidants have been identified so far. Antioxidants can be effective in three known ways to prevent and treat cancer: 1 . destruction of free radicals; 2 . strengthening the immune system to destroy cancer cells; 3 preventing the adhesion of cancer cells to other cells and preventing their proliferation [53-57].

In the current research, the cytotoxicity of carpachromene was explored by studying its interaction with normal (HUVEC) and a common human ovarian cancer cell line, i.e. SW 626, by MTT assay for $48 \mathrm{~h}$. The interactions expressed as cell viability (\%) were observed at different carpachromene concentrations $(0-1000 \mu \mathrm{g} / \mathrm{ml})$ with the five cell lines which are shown in Figures 2 and 3.

In all cases, the \% cell viability decreased with increasing carpachromene concentrations. The

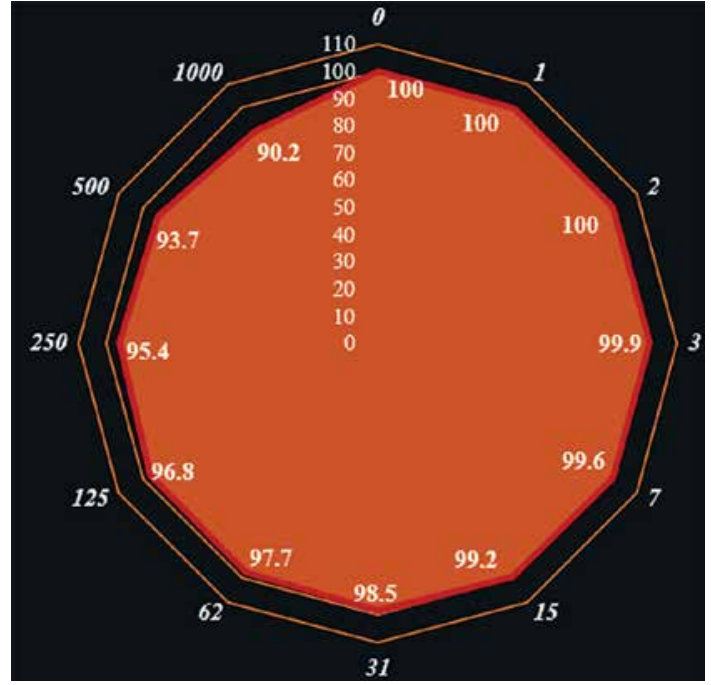

Figure 2. Cell viability of carpachromene against normal (HUVEC) cell line

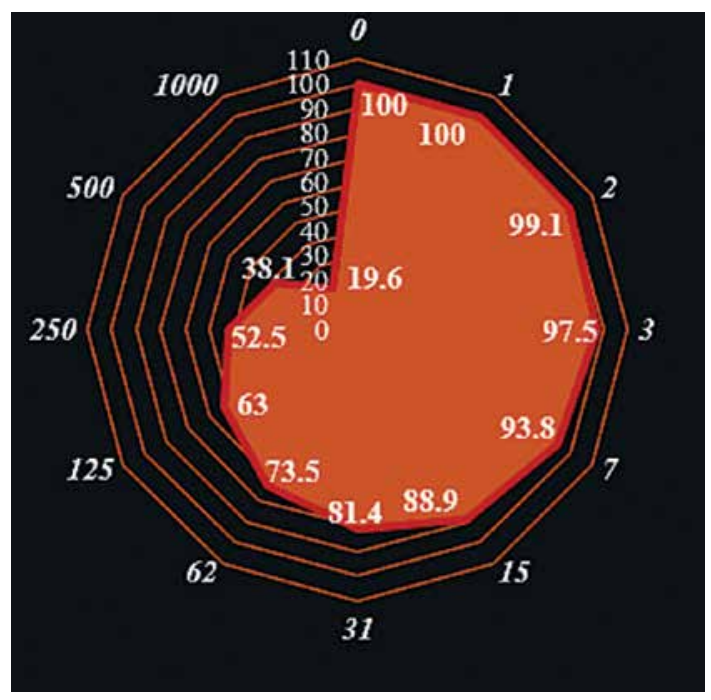

Figure 3. Anti-human ovarian cancer properties (cell viability (\%)) of carpachromene (concentrations of $0-1000 \mu \mathrm{g} / \mathrm{ml}$ ) against common human ovarian cancer cell line SW 626. The numbers indicate the percent of cell viability in the concentrations of $0-1000 \mu \mathrm{g} / \mathrm{ml}$ of carpachromene against several human ovarian cancer cell lines

$I C_{50}$ values of carpachromene against the common human ovarian cancer cell line SW 626 were 269, 98,106 , and $270 \mu \mathrm{g} / \mathrm{ml}$, respectively (Table III).

We then examined whether the release of Smac is necessary for apoptosis in ovarian cancer cells using the SW 626 cell line. We first examined mitochondrial and cytosolic Smac levels after carpachromene treatment. As shown, exposure of ovarian cancer cells to carpachromene decreased mitochondrial Smac and increased cytosolic Smac levels in a time-dependent fashion. Similar results were found for cyt c release. The release of Smac or cyt $c$ in apoptosis was also consistently shown by immunostaining. To further study the role of Smac 
Table III. $I C_{50}$ of carpachromene in the anti-human ovarian cancer test

\begin{tabular}{|lcc|}
\hline & HUVEC & SW 626 \\
\hline $\mathrm{IC}_{50}[\mu \mathrm{g} / \mathrm{ml}]$ & - & 269 \\
\hline
\end{tabular}

in cell apoptosis, we used siRNA to knock down Smac expression. As depicted in Figure $4 \mathrm{~A}$, a decrease in Smac expression was confirmed by Western blot. Silencing of Smac significantly inhibited carpachromene-induced caspase- 3 cleavage and attenuated apoptosis in these cells (Figure $4 \mathrm{~A}$ ). Moreover, overexpression of a Smac heptapeptide (Smac-N7) enhanced carpachromene-induced cell death (Figure $4 \mathrm{~B}$ ). These results suggest that Smac, the mitochondrial apoptogenic protein, plays a prominent role in the execution of carpachromene-induced apoptosis in ovarian cancer cells.

\section{Docking results}

Biological activities and anti-oxidant properties of molecules can be examined by using theoretical calculations. These calculations are an important guide for synthesizing molecules with higher activity.

As a result of molecular docking calculations of the molecule, many parameters were obtained. Each parameter obtained gives information about many different properties of the molecule. The most important parameter among these parameters is the docking score, because the numerical value of this parameter is the most important

A

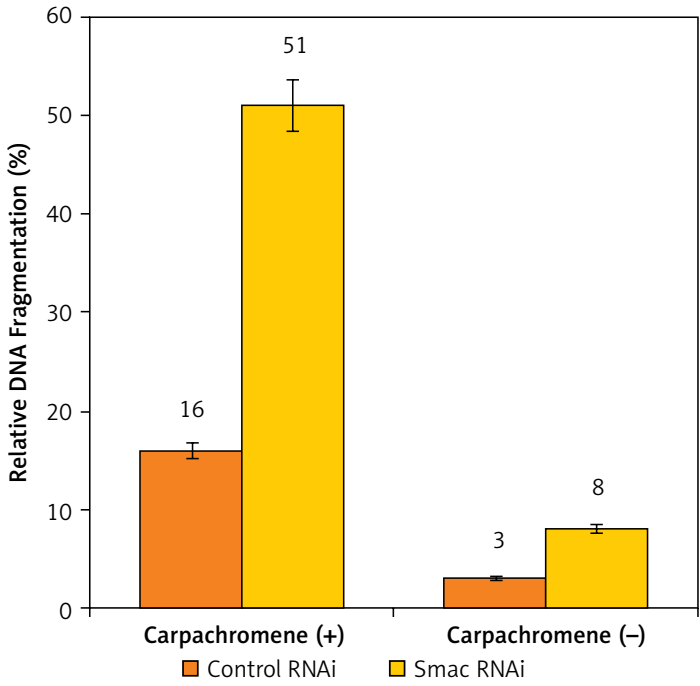

indicator of interaction between a molecule and an enzyme [58-60]. The molecule with the lowest numerical value of this parameter has the highest biological activity. The molecule with the highest biological activity is the molecule that interacts most with the enzyme. These interactions include many interactions such as hydrogen bonds, polar and hydrophobic interactions, $\pi-\pi$ and halogen [61-67]. The parameters obtained from the interaction between the molecule and the enzyme are given in Table IV and the representation of these interactions is given in Figures 5 and 6 .

Another parameter obtained from the calculations is Glide ligand efficiency. The numerical value of this parameter gives information about the activity of the molecules studied. The parameters showing the numerical values of the interaction between the molecule and the enzyme are Glide hbond, Glide evdw, Glide ecoul. On the other hand, the parameters Glide emodel, Glide energy, Glide einternal, and Glide posenum are the numerical values of the exposure formed between the molecule and the enzyme [68].

Another parameter obtained from the calculations is Glide ligand efficiency. The numerical value of this parameter gives information about the activity of the molecules studied. The parameters showing the numerical values of the interaction between the molecule and the enzyme are Glide hbond, Glide evdw, and Glide ecoul. On the other hand, Glide emodel, Glide energy, Glide einternal, and Glide posenum parameters are the numerical values of the exposure formed between the molecule and the enzyme [69].

\section{B}

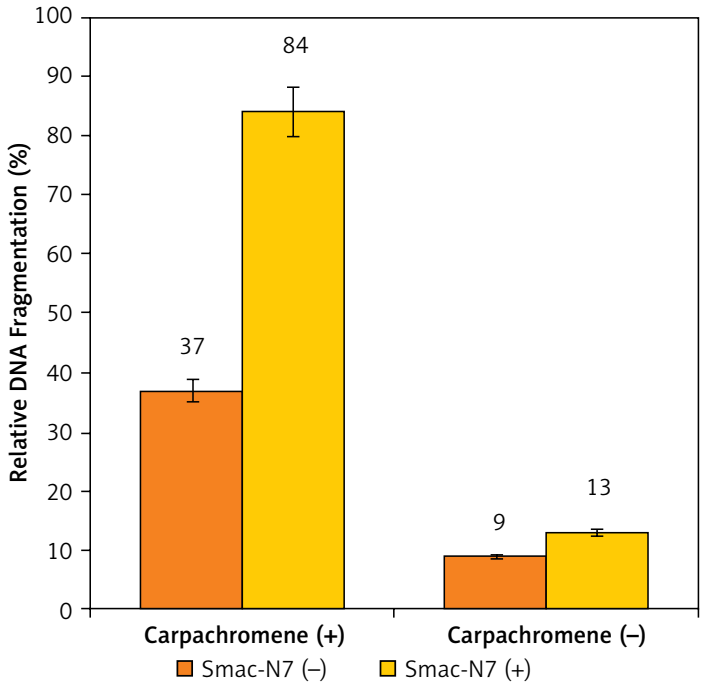

Figure 4. Smac plays an important role in carpachromene-induced apoptosis. A, cells were transfected with Smac or control (Ctrl) vector siRNA for $48 \mathrm{~h}$ and then treated with carpachromene for $24 \mathrm{~h}$. Cell apoptosis was quantitatively detected by a cell death ELISA kit as described under Material and methods. Graphs showing results of quantitative analyses. B, cells were pretreated with Smac-N7 or control $(15 \mathrm{~m})$ peptide for $3 \mathrm{~h}$, followed by treatment with or without carpachromene for $24 \mathrm{~h}$. Apoptosis was measured by a cell death ELISA kit as described under Material and methods 
Following molecular docking calculations, ADME/T analysis was performed to examine the drug-making properties of the carpachromene molecule. ADME/T analysis examines the effects and reactions of drug molecules on human metabolism. As a result of the ADME/T analysis, many parameters were obtained. With this analysis, $a b-$ sorption, distribution, metabolism, excretion and toxicity characteristics of molecules in human metabolism are predicted [8]. The parameters obtained from this analysis of the carpachromene molecule are given in Table $\mathrm{V}$.

In the ADME/T analysis, the first parameter is mol_MW, which shows the numerical value of the mol mass of the molecule. Another parameter is SASA, which is the $\pi$ (carbon and attached hydrogen) component of SASA. Another parameter is WPSA, which is the weakly polar component of SASA (halogens, P, and S). Another parameter is QPlogPo/w, which is the predicted octanol/water partition coefficient. Another parameter is CIQPlogS, which is conformation-independent predicted aqueous solubility, log S. S in $\mathrm{mol} / \mathrm{dm}^{3}$ is the concentration of the solute in a saturated solution that is in equilibrium with the crystalline
Table IV. Numerical values of the docking parameters of molecule against enzymes

\begin{tabular}{|lcc|}
\hline Variable & AChE & $\begin{array}{c}\alpha \text {-Glucosi- } \\
\text { dase }\end{array}$ \\
\hline Docking Score & -6.92 & -4.28 \\
\hline Glide ligand efficiency & -0.28 & -0.17 \\
\hline Glide hbond & 0.00 & -0.17 \\
\hline Glide evdw & -35.62 & -25.95 \\
\hline Glide ecoul & -0.39 & -4.56 \\
\hline Glide emodel & -56.03 & -43.68 \\
\hline Glide energy & -36.01 & -30.50 \\
\hline Glide einternal & 0.07 & 0.78 \\
\hline Glide posenum & 372 & 162 \\
\hline
\end{tabular}

solid. Another parameter is QPlogHERG, which is the numerical value of the estimated $I C_{50}$ value when the HERG $\mathrm{K}$ channels are blocked. Another parameter is QPPCaco, which is Caco-2 cell permeability in the gut-blood barrier for inactive transport. Another parameter is QPlogBB, which is the estimated brain/blood division coefficient. The numerical value of this parameter is for orally administered drugs; therefore, for example, do-
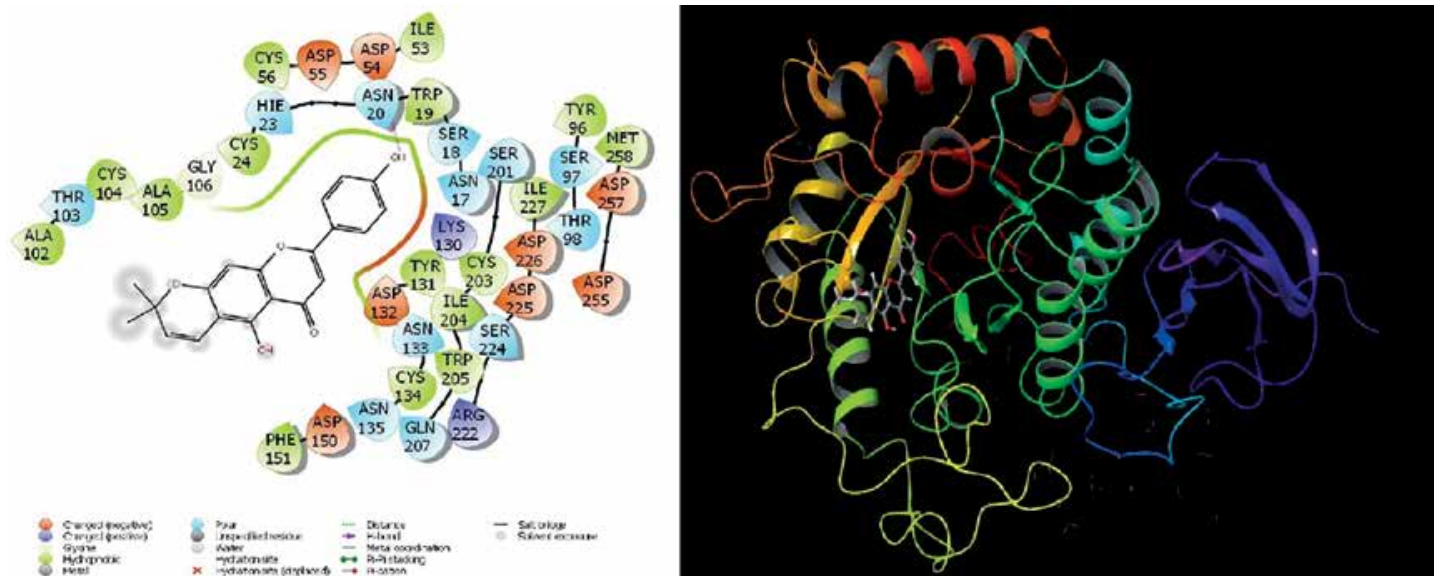

Figure 5. Presentation of interactions of carpachromene with $\alpha$-glucosidase enzyme
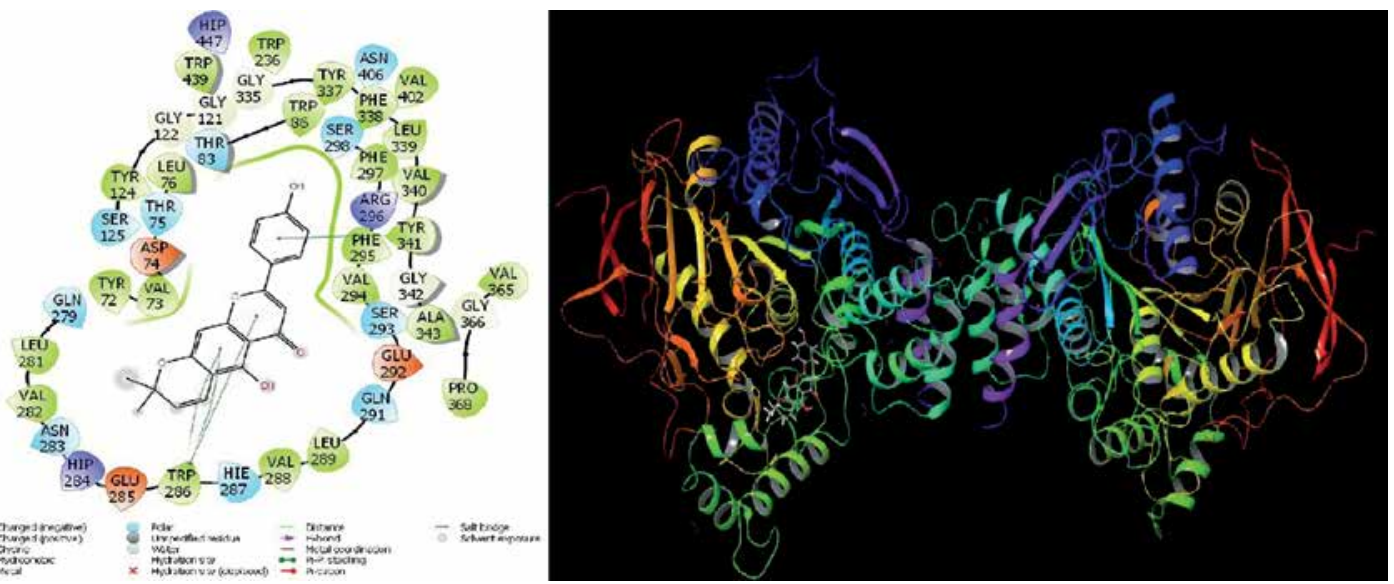

Figure 6. Presentation of interactions of carpachromene with AChE enzyme 
pamine and serotonin CNS are negative because they are molecules that are too polar to cross the blood-brain barrier. Another parameter is \#metab, which is the number of likely metabolic reactions. Another parameter is PSA, which is Van der Waals surface area of polar nitrogen and oxygen atoms [70].

The two most important parameters among all ADME/T parameters are RuleOfFive [71] and

Table V. ADME properties of molecule

\begin{tabular}{|c|c|c|}
\hline Variable & Carpachromene & Reference range \\
\hline Mol_MW & 336 & $130-725$ \\
\hline Dipole (D) & 5.55 & $1.0-12.5$ \\
\hline SASA & 606 & $300-1000$ \\
\hline FOSA & 149 & $0-750$ \\
\hline FISA & 138 & $7-330$ \\
\hline PISA & 319 & $0-450$ \\
\hline WPSA & 0 & $0-175$ \\
\hline Volume $\left(A^{3}\right)$ & 1049 & $500-2000$ \\
\hline DonorHB & 1 & $0-6$ \\
\hline AccptHB & 3.75 & $2.0-20.0$ \\
\hline $\begin{array}{l}\text { Glob (Sphere } \\
=1 \text { ) }\end{array}$ & 0.82 & $0.75-0.95$ \\
\hline QPpolrz $\left(\mathrm{A}^{3}\right)$ & 37.60 & $13.0-70.0$ \\
\hline QPlogPC16 & 11.38 & $4.0-18.0$ \\
\hline QPlogPoct & 16.43 & $8.0-35.0$ \\
\hline QPlogPw & 9.00 & $4.0-45.0$ \\
\hline QPlogPo/w & 3.69 & $-2.0-6.5$ \\
\hline QPlogS & -5.62 & $-6.5-0.5$ \\
\hline CIQPlogs & -5.68 & $-6.5-0.5$ \\
\hline QPlogHERG & -5.89 & (corcern below -5$)$ \\
\hline $\begin{array}{l}\text { QPPCaco }(\mathrm{nm} / \\
\mathrm{sec})\end{array}$ & 488 & * \\
\hline QPlogBB & -0.91 & $-3.0-1.2$ \\
\hline $\begin{array}{l}\text { QPPMDCK } \\
(\mathrm{nm} / \mathrm{sec})\end{array}$ & 228 & * \\
\hline QPlogKp & -2.75 & $\mathrm{Kp}$ in $\mathrm{cm} / \mathrm{h}$ \\
\hline IP (ev) & 9.28 & $7.9-10.5$ \\
\hline $\mathrm{EA}(\mathrm{eV})$ & 0.65 & $-0.9-1.7$ \\
\hline \#metab & 2 & $1-8$ \\
\hline QPlogKhsa & 0.66 & $-1.5-1.5$ \\
\hline $\begin{array}{l}\text { Human Oral } \\
\text { Absorption }\end{array}$ & 3 & - \\
\hline $\begin{array}{l}\text { Percent } \\
\text { Human Oral } \\
\text { Absorption }\end{array}$ & 97 & $* *$ \\
\hline PSA & 85 & $7-200$ \\
\hline RuleOfFive & 0 & Maximum is 4 \\
\hline RuleOfThree & 0 & Maximum is 3 \\
\hline $\mathrm{Jm}$ & 0.00 & - \\
\hline
\end{tabular}

RuleOfThree [72]. The numerical value of these two parameters is expected to be zero. The RuleOfFive parameter is also the fifth rule of Lipinski's Pfizer. The rules are: $\mathrm{mol} M W<500$, QPlogP $\mathrm{o} / \mathrm{w}<5$, donorHB $\leq 5$, accptHB $\leq 10$. However, the RuleOfThree parameter is known as Jorgensen's rule of three. The three rules are: QPlogS $>-5.7$, $\mathrm{QP}$ PCaco $>22 \mathrm{~nm} / \mathrm{s}$, \#Primary Metabolites $<7$. If the numerical value of the RuleOfThree parameter is zero, this molecule can be used orally as a medicine. The last and another important parameter is $\mathrm{Jm}$, which is the predicted maximum transdermal transport rate, $\mathrm{Kp} \times \mathrm{MW} \times \mathrm{S}\left(\mu \mathrm{g} \times \mathrm{cm}^{-2} \times \mathrm{h}^{-1}\right) . \mathrm{Kp}$ and $S$ are obtained from the aqueous solubility and skin permeability, QPlogKp and QPlogS. The theoretical estimate obtained by applying molecules that can be drugs with this parameter to the skin are numerical values (Table VI) [72].

For the anti-oxidant calculations of the molecule, removal of hydrogen from the $\mathrm{OH}$ bond in the molecule was done. The carpachromene molecule has $2 \mathrm{OH}$ bonds. One is oxygen atom 24 and the other is oxygen atom 8 . The numerical value of $\mathrm{BDE}$ for the $\mathrm{OH}$ bond of the carpachromene molecule is a parameter related to the HAT mechanism. The molecule with the numerical value of the smaller BDE parameter was found to have higher radical-scavenging activity.

In the first step of the SET-PT mechanism, the IP value of the carpachromene molecule is calculated. If a molecule has a lower IP value, it is known that the molecule has a higher ability to give electrons. In the second step of the SET-PT mechanism, PDE values are calculated. If a molecule has a lower PDE value, this means an easier reaction. Finally, the SPLET mechanism consists of two stages. In the first stage, the PA parameter is calculated. If a molecule has a lower PA value, it indicates that the molecule has a higher proton affinity. In the second stage of SPLET, the ETE parameter is calculated. If a molecule has a lower ETE value, that molecule indicates an easier reaction $[71,72]$.

The parameters of all calculated anti-oxidant properties are given in Table VI. The parameters given in this table are calculated both in gas phase and water phase. The calculations were made for two $\mathrm{O}-\mathrm{H}$ bonds, but different positions were more active in both phases. The main reason for this is that the solvent affects the stability of the formed phenolate anion [72].

As a result of the calculations made, spin densities were calculated to examine the charge density of the molecules. Spin density values of the molecule are given above the atoms in Figure 7. The lower spin density shown on the atoms in the molecule indicates greater delocalization. Consequently, higher delocalization means easier radical formation. Being more radical as a result of all 
Table VI. Anti-oxidant properties of molecule

\begin{tabular}{|lccccc|}
\hline In gas phase & BDE & IP & PDE & PA & ETE \\
\hline O8-H & 53.15 & 147.89 & 219.76 & 288.67 & 78.98 \\
\hline O24-H & 40.18 & 147.89 & 206.78 & 284.45 & 70.22 \\
\hline In water phase & & & & \\
\hline O8-H & 51.93 & 113.52 & 252.91 & 301.81 & 64.63 \\
\hline O24-H & 93.21 & 113.52 & 294.19 & 297.59 & 110.12 \\
\hline
\end{tabular}
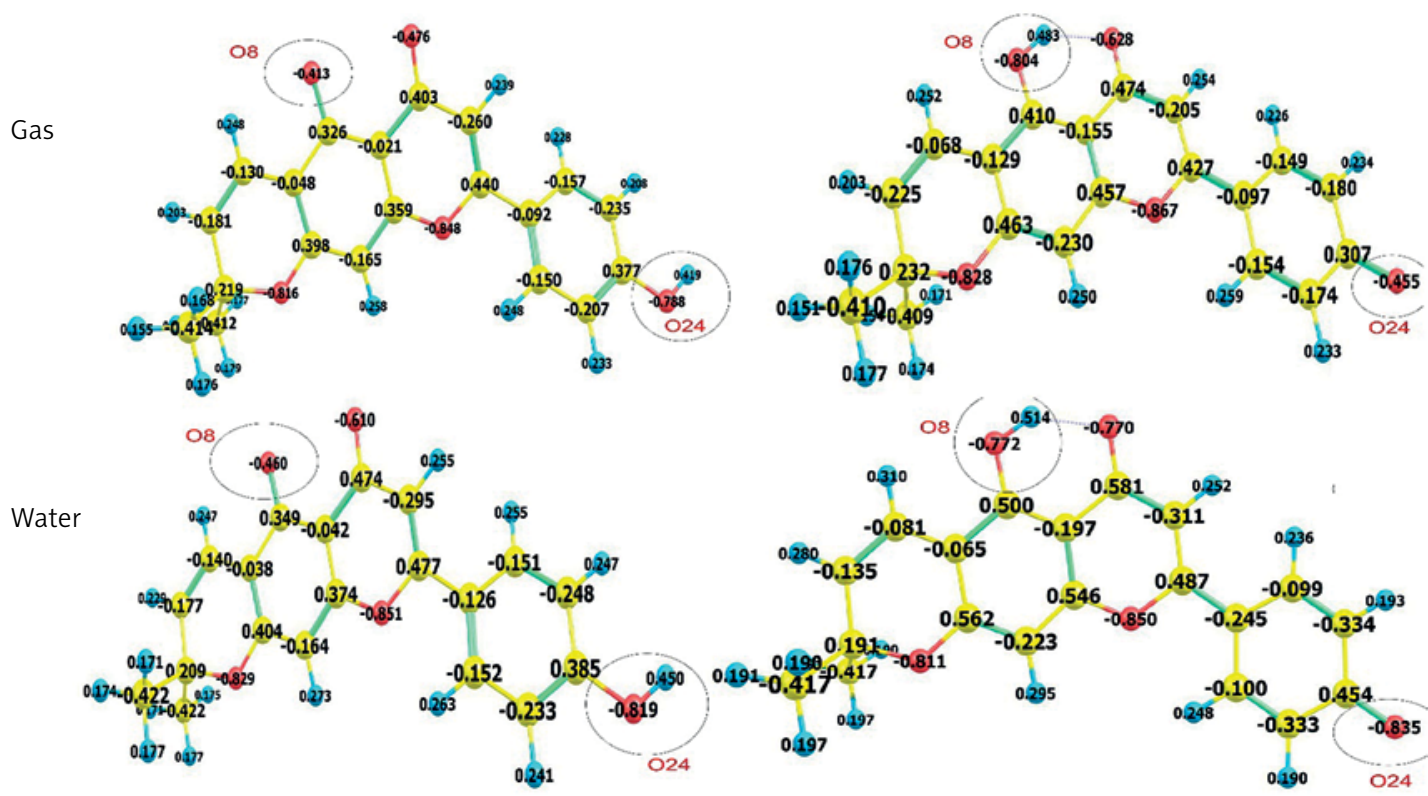

Figure 7. Spin density distributions of molecule

these situations affects the stability of the radical and causes the formation of more stable radicals.

In conclusion, biological activities of the carpachromene molecule against many enzymes were compared in molecular docking and anti-oxidant properties. Many parameters were obtained for the biological activity of the carpachromene molecule against enzymes. Afterwards, ADME/T analysis of this molecule was done. The ability of the carpachromene molecule to be used as a drug in the future was examined. These studies will be a great guide for future in vivo and in vitro studies. Finally, the anti-oxidant properties of the carpachromene molecule were examined. Necessary parameters for radical activity were collected.

Carpachromene was also assessed in biological applications such as radical scavenging, cytotoxicity, and anticancer (adenocarcinoma) activities. Carpachromene exhibited good antioxidant properties, even better than the reference standard molecule. It also showed significant cytotoxic activities against the common human ovarian cancer cell line SW 626.

\section{Acknowledgments}

Yunjing Song and Jian Wang are the co-first author.
The project was supported by a Science and Technology project of Jiangxi Health Committee (Smac. Caspases9 signaling pathways promote apoptosis of epithelial ovarian cancer cells) Project No: 202140738.

\section{Conflict of interest}

The authors declare no conflict of interest.

\section{References}

1. Murray AP, Faraoni MB, Castro MJ, Alza NP, Cavallaro V. Natural AChE inhibitors from plants and their contribution to Alzheimer's disease therapy. Curr Neuropharmacol 2013; 11: 388-413.

2. Tundis R, Bonesi M, Menichini F, Loizzo MR. Recent knowledge on medicinal plants as source of cholinesterase inhibitors for the treatment of dementia. Med Chem 2016; 16: 8.

3. Rasouli H, Hosseini-Ghazvini SMB, Adibi H, Khodarahmi R. Differential $\alpha$-amylase/ $\alpha$-glucosidase inhibitory activities of plant-derived phenolic compounds: a virtual screening perspective for the treatment of obesity and diabetes. Food Funct 2017; 8: 1942-54.

4. Kim JH, Cho CW, Kim HY, et al. $\alpha$-Glucosidase inhibition by prenylated and lavandulyl compounds from Sophora flavescens roots and in silico analysis. Int J Biol Macromol 2017; 102: 960-9. 
5. Sayin K, Kariper SE, Sayin TA, Karakas D. Theoretical spectroscopic study of seven zinc (II) complex with mac rocyclic Schiff-base ligand. Spectrochim Acta Part A Mol Biomol Spectrosc 2014; 133: 348-56.

6. Sagdinc SG, Erdas D, Gunduz I, Sahinturk AE. FT-IR and FT-Raman spectra, molecular structure and first-order molecular hyperpolarizabilities of a potential antihistaminic drug, cyproheptadine $\mathrm{HCl}$. Spectrochim Acta Part A Mol Biomol Spectrosc 2015; 134: 350-60.

7. Soayed AA, El-Husseiny AF. Potentiometry and geometrical structure of some azodye compounds and their metal complexes. J Mol Liq 2015; 209: 258-66.

8. Mahmoud WH, Mahmoud NF, Mohamed GG, El-Bindary AA, El-Sonbati AZ. Supramolecular structural, thermal properties and biological activity of 3-(2-methoxyphenoxy) propane-1, 2-diol metal complexes. J Mol Struct 2015; 1086: 266-75.

9. El-Sonbati AZ, Diab MA, El-Bindary AA, Ghoneim MM, Mohesien MT, El-Kader MA. Polymeric complexes - LXI. Supramolecular structure, thermal properties, SS-DNA binding activity and antimicrobial activities of polymeric complexes of rhodanine hydrazone compounds. J Mol Liq 2016; 215: 711-39.

10. Koç E, Üngördü A, Candan F. Antioxidant properties of methanolic extract of 'Veronica multifida'and DFT and $\mathrm{HF}$ analyses of its the major flavonoid component. J Mol Structure 2019; 1197: 436-42.

11. Kamkar A, Javan AJ, Asadi F, Kamalinejad M. The antioxidative effect of Iranian Mentha pulegium extracts and essential oil in sunflower oil. Food Chem Toxicol 2010; 48: $1796-800$

12. Carling T, Udelsman R. Thyroid cancer. Ann Rev Med 2014; 65: 125-37.

13. GBD 2015 Disease and Injury Incidence and Prevalence, Collaborators. Global, regional, and national incidence, prevalence, and years lived with disability for 310 diseases and injuries, 1990-2015: a systematic analysis for the Global Burden of Disease Study 2015. Lancet 2016; 388: 1545-602.

14. Durante C, Grani G, Lamartina L, Filetti S, Mandel SJ, Cooper DS. The diagnosis and management of thyroid nodules. JAMA 2018; 319: 914-24.

15. GBD 2015 Mortality and Causes of Death, Collaborators Global, regional, and national life expectancy, all-cause mortality, and cause-specific mortality for 249 causes of death, 1980-2015: a systematic analysis for the Global Burden of Disease Study 2015. Lancet 2016; 388: 1459 544.

16. Moschini M, Simone G, Stenzl A, Gill IS, Catto J. Critical review of outcomes from radical cystectomy: can complications from radical cystectomy be reduced by surgical volume and robotic surgery? Eur Urol Focus 2016; 2: 19-29.

17. Singh R, Lillard JW. Nanoparticle-based targeted drug delivery. Exp Mol Pathol 2009; 86: 215-23.

18. Hemmati S, Joshani Z, Zangeneh A, et al. Biosynthesis and chemical characterization of polydopamine-capped silver nanoparticles for the treatment of acute myeloid leukemia in comparison to doxorubicin in a leukemic mouse model. Appl Organometal Chem 2020; 34: e5277.

19. Mohammadi G, Zangeneh MM, Zangeneh A, et al. Chemical characterization and anti-breast cancer effects of silver nanoparticles using Phoenix dactylifera seed ethanolic extract on 7,12-Dimethylbenz[a] anthracene-induced mammary gland carcinogenesis in Sprague Dawley male rats. Appl Organometal Chem 2020; 34: e5136.
20. Zangeneh MM, Bovandi S, Gharehyakheh S, et al Green synthesis and chemical characterization of silver nanoparticles obtained using Allium saralicum aqueous extract and survey of in vitro antioxidant, cytotoxic, antibacterial and antifungal properties. Appl Organometal Chem 2019; 33: e4961.

21. Hamelian M, Zangeneh MM, Shahmohammadi A, et al. Pistacia atlantica leaf extract mediated synthesis of silver nanoparticles and their antioxidant, cytotoxicity, and antibacterial effects under in vitro condition. Appl Organometal Chem 2020; 34: e5278.

22. Hemmati S, Rashtiani A, Zangeneh MM, et al. Green synthesis and characterization of silver nanoparticles using Fritillaria flower extract and their antibacterial activity against some human pathogens. Polyhedron 2019; 158: 8-14.

23. Zangeneh MM, Joshani Z, Zangeneh A, et al. Green synthesis of silver nanoparticles using aqueous extract of Stachys lavandulifolia flower, and their cytotoxicity, antioxidant, antibacterial and cutaneous wound-healing properties. Appl Organometal Chem 2019; 33: e5016.

24. Zangeneh A, Zangeneh MM, Moradi R. Ethnomedicinal plant-extract-assisted green synthesis of iron nanoparticles using Allium saralicum extract, and their antioxidant, cytotoxicity, antibacterial, antifungal and cutaneous wound-healing activities. Appl Organometal Chem 2019; 33: e5247.

25. Schrodinger L. Small-Molecule Drug Discovery Suite 2019-4; 2019.

26. Schrödinger Release 2019-4: Protein Preparation Wizard; Epik, Schrödinger, LLC, New York, NY, 2016; Impact, Schrödinger, LLC, New York, NY, 2016; Prime, Schrödinger, LLC, New York, NY, 2019.

27. Friesner RA, Murphy RB, Repasky MP, et al. Extra precision glide: Docking and scoring incorporating a model of hydrophobic enclosure for protein-ligand complexes. J Med Chem 2006; 49: 6177-96.

28. Sastry GM, Adzhigirey M, Day T, Annabhimoju R, Sherman W. Protein and ligand preparation: parameters, protocols, and influence on virtual screening enrichments. J Computer Aided Mol Design 2013; 27: 221-34

29. Schrödinger Release 2019-4: LigPrep, Schrödinger, LLC, New York, NY, 2019.

30. Du Q, Qian Y, Yao X, Xue W. Elucidating the tight-binding mechanism of two oral anticoagulants to factor $X a$ by using induced-fit docking and molecular dynamics simulation. J Biomol Structure Dynamics 2020; 38: 625-33.

31. Golubev AM, Nagem RAP, Neto JB, et al. Crystal structure of $\alpha$-galactosidase from Trichoderma reesei and its complex with galactose: implications for catalytic mechanism. J Mol Biol 2004; 339: 413-22.

32. Cheung J, Gary EN, Shiomi K, Rosenberry TL. Structures of human acetylcholinesterase bound to dihydrotanshinone I and territrem B show peripheral site flexibility. ACS Med Chem Letters 2013; 4: 1091-6.

33. Alterio V, Monti SM, Truppo E, Pedone C, Supuran CT, De Simone $G$. The first example of a significant active site conformational rearrangement in a carbonic anhydrase-inhibitor adduct: the carbonic anhydrase 1-topiramate complex. Organic Biomol Chem 2010; 8: 3528-33.

34. Ivanova J, Leitans J, Tanc $M$, et al. X-ray crystallography-promoted drug design of carbonic anhydrase inhibitors. Chem Commun 2015; 51: 7108-11.

35. Schrödinger Release 2020-1: QikProp, Schrödinger, LLC, New York, NY, 2020

36. Marković Z, Jeremić S, Marković JD, Pirković MS, Amić D. Influence of structural characteristics of substituents 
on the antioxidant activity of some anthraquinone derivatives. Computational Theoretical Chemistry 2016; 1077: 25-31.

37. Sari S, Barut B, Özel A, Şöhretoğlu D. Tyrosinase inhibitory effects of Vinca major and its secondary metabolites: enzyme kinetics and in silico inhibition model of the metabolites validated by pharmacophore modelling. Bioorganic Chem 2019; 92: 103259.

38. Koç E, Üngördü A, Candan F. Antioxidant properties of methanolic extract of 'Veronica multifida'and DFT and $\mathrm{HF}$ analyses of its the major flavonoid component. J Mol Structure 2019; 1197: 436-42.

39. Urbaniak A, Szeląg M, Molski M. Theoretical investigation of stereochemistry and solvent influence on antioxidant activity of ferulic acid. Computational Theoretical Chemistry 2013; 1012: 33-40.

40. Kheirabadi R, Izadyar M. Antioxidant activity of selenenamide-based mimic as a function of the aromatic thiols nucleophilicity, a DFT-SAPE model. Computational Biol Chem 2018; 75: 213-21.

41. Dennington R, Keith T, Millam J. GaussView, Version 6, Semichem Inc., Shawnee Mission, KS 2016.

42. Vautherin D, Brink DM. Hartree-Fock calculations with skyrme's interaction. I. Spherical nuclei. Phys Rev C 1972; 5; 626-47.

43. Becke AD. Density-functional thermochemistry. III. The role of exact exchange. J Chem Phys 1993; 98: 5648-52.

44. Ellman GL, Courtney KD, Andres Jr V, Featherstone RM. A new and rapid colorimetric determination of acetylcholinesterase activity. Biochem Pharmacol 1961; 7: 88-95.

45. Szwajgier D, Borowiec K. Phenolic acids from malt are efficient acetylcholinesterase and butyrylcholinesterase inhibitors. J Inst Brew 2012; 118: 40-8.

46. Muhammad A, Odunola OA, Gbadegesin MA, Sallau AB, Ndidi US, Ibrahim MA. Inhibitory effects of sodium arsenite and acacia honey on acetylcholinesterase in rats. Int J Alzheimer's Dis 2015; 2015: 903603.

47. Tao Y, Zhang Y, Cheng Y, Wang Y. Rapid screening and identification of $\alpha$-glucosidase inhibitors from mulberry leaves using enzyme-immobilized magnetic beads coupled with HPLC/MS and NMR. Biomed Chromatography 2013; 27: 148-55.

48. Lee BH, Rose DR, Lin AHM, Quezada-Calvillo R, Nichols BL, Hamaker BR. Contribution of the individual small intestinal $\alpha$-glucosidases to digestion of unusual $\alpha$-linked glycemic disaccharides. J Agric Food Chem 2016; 64: 6487-94.

49. Lineweaver $H$, Burk D. The determination of enzyme dissociation constants. J Am Chem Soc 1934; 56: 658-66.

50. Zangeneh MM, Saneei S, Zangeneh A, et al. Preparation, characterization, and evaluation of cytotoxicity, antioxidant, cutaneous wound healing, antibacterial, and antifungal effects of gold nanoparticles using the aqueous extract of Falcaria vulgaris leaves. Appl Organometal Chem 2019; 33: e5216.

51. Hemmati S, Joshani Z, Zangeneh A, et al. Green synthesis and chemical characterization of Thymus vulgaris leaf aqueous extract conjugated gold nanoparticles for the treatment of acute myeloid leukemia in comparison to doxorubicin in a leukemic mouse model. Appl Organometal Chem 2020; 34: e5267.

52. Ahmeda A, Zangeneh A, Zangeneh MM. Green synthesis and chemical characterization of gold nanoparticle synthesized using Camellia sinensis leaf aqueous extract for the treatment of acute myeloid leukemia in comparison to daunorubicin in a leukemic mouse model. Appl Organometal Chem 2020; 34: e5290.
53. Katata-Seru L, Moremedi T, Aremu OS, et al. Green synthesis of iron nanoparticles using Moringa oleifera extracts and their applications: Removal of nitrate from water and antibacterial activity against Escherichia coli. J Mol Liq 2018; 256: 296-304.

54. Sangami S, Manu M. Synthesis of green iron nanoparticles using Laterite and their application as a Fenton-like catalyst for the degradation of herbicide Ametryn in water. Environ Technol Innov 2017; 8: 150-63.

55. Beheshtkhoo N, Kouhbanani MAJ, Savardashtaki A, et al. Green synthesis of iron oxide nanoparticles by aqueous leaf extract of Daphne mezereum as a novel dye removing material. Appl Phys A 2018; 124: 363-9.

56. Radini IA, Hasan N, Malik MA, et al. Biosynthesis of iron nanoparticles using Trigonella foenum-graecum seed extract for photocatalytic methyl orange dye degradation and antibacterial applications. J Photochem Photobiol B 2018; 183: 154-63.

57. Oganesvan G, Galstyan A, Mnatsakanyan V, et al. Phenolic and flavonoid compounds of Ziziphora clinopodioides. Chem Nat 1991; 27: 247-7.

58. El-Bindary AA, Ghoneim MM, Diab MA, El-Sonbati AZ, Serag LS. Thermodynamic studies of $\mathrm{N}$-allylrhodanine derivatives and their metal complexes. J Mol Liq 2016; 223: 448-61.

59. Sayin K, Kurtoglu N, Kose M, Karakas D, Kurtoglu M. Computational and experimental studies of 2-[(E)-hydrazinylidenemethyl]-6-methoxy-4-[(E)-phenyldiazenyl] phenol and its tautomers. J Mol Struct 2016; 1119: 413-22.

60. Allouche AR. Gabedit - a graphical user interface for computational chemistry softwares. J Comput Chem 2011; 32: 174-82

61. Sayin K, Üngördü A. Investigations of structural, spectral and electronic properties of enrofloxacin and boron complexes via quantum chemical calculation and molecular docking. Spectrochim Acta A Mol Biomol Spectroscopy 2019; 220: 117102.

62. Sayin K, Karakaş D. Investigation of structural, electronic properties and docking calculations of some boron complexes with norfloxacin: a computational research. Spectrochim Acta A Mol Biomol Spectroscopy 2018; 202: 276-83.

63. Sayin K, Karakaş D. Quantum chemical investigation of levofloxacin-boron complexes: a computational approach. J Mol Structure 2018; 1158: 57-65.

64. Sayin K, Üngördü A. Investigation of anticancer properties of caffeinated complexes via computational chemistry methods. Spectrochim Acta A Mol Biomol Spectroscopy 2018; 193: 147-55.

65. Sayin K, Karakaş D. Determination of structural, spectral, electronic and biological properties of tosufloxacin boron complexes and investigation of substituent effect. J Mol Structure 2017; 1146: 191-7.

66. Jayarajan R, Satheeshkumar R, Kottha T, Subbaramanian S, Sayin K, Vasuki G. Water mediated synthesis of 6-amino-5-cyano-2-oxo-N-(pyridin-2-yl)-4-(p-tolyl)$2 \mathrm{H}$-[1, 2'-bipyridine]-3-carboxamide and 6-amino-5cyano-4-(4-fluorophenyl)-2-oxo-N-(pyridin-2-yl)-2H-[1, 2'-bipyridine]-3-carboxamide-An experimental and computational studies with non-linear optical (NLO) and molecular docking analyses. Spectrochim Acta A Mol Biomol Spectroscopy 2020; 229: 117861.

67. Üngördü A, Sayin K. Quantum chemical calculations on sparfloxacin and boron complexes. Chem Physics Letters 2019; 733: 136677.

68. Gill SS, Anderson GM, Fischer HD, et al. Syncope and its consequences in patients with dementia receiving cho- 
linesterase inhibitors: a population-based cohort study. Arch Intern Med 2009; 169: 867-73.

69. Ali TB, Schleret TR, Reilly BM, Chen WY, Abagyan R. Adverse effects of cholinesterase inhibitors in dementia, according to the pharmacovigilance databases of the United-States and Canada. PLoS One2015; 10: e0144337.

70. Lipinski CA. Lead-and drug-like compounds: the rule-offive revolution. Drug Discov Today Technologies 2004; 1: 337-41.

71. Lipinski CA, Lombardo F, Dominy BW, Feeney PJ. Experimental and computational approaches to estimate solubility and permeability in drug discovery and development settings. Adv Drug Deliv Rev 1997; 23: 3-25.

72. Jorgensen WJ, Duffy EM. Prediction of drug solubility from structure. Adv Drug Deliv Rev 2002; 54: 355-66. 